\title{
Instrumentos de inteligência e interesses em orientação profissional
}

\author{
Silvia Godoy \\ Ana Paula Porto Noronha \\ Rodolfo Augusto Matteo Ambiel \\ Maiana Farias Oliveira Nunes \\ Universidade São Francisco
}

\begin{abstract}
Resumo
O uso de instrumentos psicometricamente ajustados ao contexto em que serão utilizados é fundamental para a confiabilidade de processos de avaliação psicológica. Em situações de orientação profissional, tal preceito tem igual importância, uma vez que a análise de diferentes construtos deve levar o sujeito a melhor se conhecer, proporcionando uma escolha adequada da profissão. O objetivo desse estudo foi correlacionar diferentes instrumentos de avaliação de interesses profissionais e inteligência, aplicados a 312 participantes de um programa de orientação profissional, com média de idade de 16,13 anos $(D P=2,07)$. Os resultados mostraram algumas correlações significativas entre BPR-5 e LIP, por um lado, e entre o INV e Angelini de outro. Novos estudos com amostras maiores e mais heterogêneas geograficamente são sugeridos, a fim de se obter mais evidências de validade, principalmente para os instrumentos ainda não aprovados pelo Conselho Federal de Psicologia.
\end{abstract}

Palavras-chave: avaliação psicológica; interesses profissionais; inteligência; validade

\begin{abstract}
Intelligence and interests tests in vocational guidance. The use of psychometrically adjusted instruments to the context in which they will be used is important to trust the psychological assessments process. In professional counseling situations, such use has the same importance, since different constructs analyses must provide a better self-knowledge, facilitating an adequate professional choice. The goal of this study was to correlate different intelligence and vocational interests instruments, answered by 312 students from a vocational counseling process, with age average 16.13 years old $(S D=2.07)$. Results showed significant correlations between BPR-5 and LIP, in one hand, and between INV and Angelini, in the other. New studies with larger and more geographically heterogeneous samples are suggested, aiming to get more validity evidences, mainly to the not approved instruments by the Federal institution that regulates psychological test use.
\end{abstract}

Keywords: psychological assessment; professional interests; intelligence; validity

$\mathrm{O}$ S serviços psicológicos dedicados à avaliação de carreira possuem dois focos principais, um voltado para a pessoa e outro para o problema. Mais especificamente, podem, por um lado, auxiliar o interessado a refletir sobre características pessoais que estejam associadas a profissões (como interesses e habilidades) ou, por outro lado, intervir sobre os processos psicológicos que atrapalham a tomada de decisão sobre a carreira, a exemplo da indecisão profissional, ansiedade e outros (Savickas, 2004). Uma das modalidades de serviços de carreira é a Orientação Profissional (OP), que tem como principal finalidade auxiliar os indivíduos com dúvidas no que se refere à carreira profissional a avaliar características pessoais, com vistas a realizar escolhas profissionais (Savickas, 1999, 2004). O processo de OP, essencialmente, ajuda que as pessoas identifiquem suas preferências, obtenham informações sobre as diferentes áreas profissionais e explorem suas escolhas
(Holland, 1977).

No contexto brasileiro, de acordo com Melo-Silva, Lassance e Soares (2004), a Orientação Profissional é compreendida como a ajuda para a tomada de decisão em momentos específicos, como a passagem de um ciclo educativo a outro; a transição dos estudos ao mundo do trabalho; mudança de ocupação ou emprego ou preparação e adaptação para a aposentadoria. A intervenção ocorre, portanto, em momentos críticos da trajetória profissional de pessoas e grupos. Ainda com relação aos serviços de orientação profissional, outros autores destacam a importância de analisar métodos e instrumentos psicológicos utilizados nesse contexto, de modo a disponibilizar serviços adequados, fundamentados teórica e empiricamente (Melo-Silva, Oliveira, \& Coelho, 2002).

Nesse sentido, no que respeita aos métodos e técnicas, a Orientação Profissional foi influenciada pela psicometria, 
compreendida como a ciência da medida e da mensuração, trazendo benefícios como a construção de instrumentos de avaliação. Para Anastasi e Urbina (2000), o instrumento adequado é aquele que respeita os padrões psicométricos com o intuito de gerar resultados mais confiáveis e precisos. No que se refere à construção e validação de testes para uso em OP no Brasil, há a necessidade de maior incremento nas ações dos pesquisadores, já que é diminuto o número de testes que possuem parecer favorável do Conselho Federal de Psicologia (CFP, 2007), ou seja, uma pequena parte está em consonância com os padrões mínimos de adequação, no que se refere aos estudos das propriedades psicométricas e normatização com amostras brasileiras.

Tais considerações podem ser corroboradas pelo estudo de Noronha, Freitas e Ottati (2003). No que diz respeito ao uso de instrumentos para orientação profissional (OP) no Brasil, as autoras, ao analisarem oito instrumentos para este fim, ressaltaram uma carência de estudos na área, destacando a necessidade do desenvolvimento de trabalhos que visem garantir a qualidade psicométrica deles. Foram avaliados diferentes aspectos, como autor, editor, ano de publicação, padronização, validade e precisão. Os resultados indicaram a ausência de informações sobre o autor e data de publicação em alguns manuais. Com relação à padronização, $37,5 \%$ dos instrumentos consultados não ofereciam informações sobre estes procedimentos, $25 \%$ dos instrumentos não possuíam nenhum dado que indicasse estudos de validação e $62,5 \%$ dos instrumentos não apresentaram informações sobre sua precisão.

Os resultados do estudo (Noronha, Freitas, \& Otatti, 2003), no que se refere à necessidade de melhoria da qualidade dos instrumentos psicológicos utilizados em OP, estava em consonância com a proposta do Conselho Federal de Psicologia CFP (2001, 2003), na medida em que esse tem buscado estimular o aprimoramento da qualidade dos instrumentos utilizados no país, a fim de assegurar maior qualidade dos serviços prestados à população. Poucos instrumentos aprovados pelo Conselho Federal de Psicologia (CFP, 2007) possuem indicação nos manuais para a utilização em OP, quais sejam, o BBT - Teste de Fotos de Profissão (Jacquemin, 2000), o EMEP- Escala de Maturidade para a Escolha Profissional (Neiva, 1999) e o EAP (Noronha, Sisto, \& Santos, 2007). O BBT avalia os interesses profissionais, em uma abordagem psicodinâmica, mais especialmente as inclinações profissionais; o EMEP aborda a maturidade para a escolha profissional, sendo destinado à população de ensino médio. A escala é composta de 45 itens e encontra referencial nas teorias desenvolvimentistas de Super (1955) e Crites (1969), que conceituam o desenvolvimento vocacional como um processo contínuo que se estende da infância à velhice, geralmente ordenado. Por fim, o EAP avalia os interesses profissionais, tendo sido construído a partir de atividades profissionais típicas em determinados cursos de graduação, sendo indicado no manual para uso com público universitário.

Em acréscimo, outro instrumento, bastante relatado nas pesquisas, embora sem manual comercializado no Brasil, é o Self-Directed Search Career Explorer (SDS- Holland, Fritzsche, \& Powell, 1994), fundamentado na teoria dos seis tipos de personalidade vocacional de Holland (1963). A concepção teórica desenvolvida pelo autor, intitulada Modelo Hexagonal, sistematiza uma tipologia caracterizando os protótipos ou casos mais extremos de tipos profissionais. Ele propõe seis tipos básicos, a saber, Realista (R), Investigativo (I), Artístico (A), Social (S), Empreendedor (E) e Convencional (C). O modelo sugere que a congruência entre personalidade e ambiente profissional produz bons resultados, como satisfação e realização no trabalho, uma vez que os interesses profissionais estão intimamente relacionados com a personalidade do sujeito.

Nesse sentido, vale destacar o estudo de validação do SDS realizado por Mansão (2005) com amostra brasileira. A autora aplicou os instrumentos SDS, BPR 5 - Forma B (Primi \& Almeida, 1998), LIP (Del-Nero, 1984), Inventário de Interesses Angelini (s.d.) em 1161 estudantes do Ensino Médio. Os resultados revelaram que o instrumento apresenta capacidade de avaliar os interesses profissionais de forma simples e rápida, permitindo o delineamento de um perfil tipológico útil para a escolha de uma ocupação. Reafirmou, ainda, que a teoria de Holland é um importante referencial para os estudiosos da Orientação Profissional, pois permite a compreensão dos interesses profissionais, aspecto relevante da escolha, sem colocar em detrimento outros fatores atrelados ao processo como as características ambientais e de personalidade.

Como parte do estudo anterior, Mansão (2005) correlacionou o SDS e o LIP, cujos resultados apresentaram correlações significativas entre os tipos do SDS e as áreas de interesse profissional do LIP. No tipo Realista, as correlações foram significativas com as áreas das Ciências Físicas (CF) e do Cálculo (C). O tipo Investigativo apresentou o perfil por ocupações predominantemente na área das ciências da saúde e pesquisa, relacionada com as áreas Ciências Biológicas (CB) e Ciências Físicas (CF). No tipo Artístico, as correlações mais significativas foram com as áreas Artístico (A), Lingüístico (L) e Social (S). Ainda, o tipo Social apresentou correlação significativa com a área de interesses CB (Ciências Biológicas). Essa associação é encontrada em sujeitos cujo interesse está direcionado para atividades profissionais que envolvam relação direta de ajuda, de contato com pessoas por meio de ocupações da área da saúde como Enfermagem, Fisioterapia, Terapia Ocupacional, Psicologia, justificando-se assim, a combinação "Social e Ciências Biológicas". O tipo Empreendedor obteve correlações negativas com a área Ciências Biológicas (CB), ou seja, esse tipo refere-se a indivíduos cujo interesse está centrado em atividades de comando, gerência, persuasão que se distanciam das atividades da área Ciências Biológicas (CB) que se referem às atividades de investigar, pesquisar, entender fenômenos científicos entre outras.

A área Burocrática Administrativa (BA) e Persuasiva (P), que expressam respectivamente interesses por profissões ligadas a administrar, comandar, organizar e argumentar, apresentaram os maiores índices de correlação com o tipo Empreendedor que, conforme definição de Holland, refere-se a indivíduos que gostam de assuntos ligados a política e economia, demonstra a preocupação com o poder, posição social, liderança e procuram, por meio de sua profissão, satisfazer suas necessidades de comando. O tipo Convencional se correlacionou com as áreas 
Burocrática Administrativa e Cálculo. Entre as ocupações que podem representar essa combinação aparecem contabilidade, administração, bancário, economista, (mais diretamente relacionadas a administrar e calcular) e biblioteconomia, almoxarifado (mais relacionadas a organizar, sistematizar), de acordo com os dados apresentados por Mansão (2005).

Além dos interesses, outra informação que pode ser útil no processo de OP são as habilidades cognitivas ou a inteligência. É possível, por exemplo, comparar os resultados de uma pessoa com o de pares (avaliação inter-sujeitos) ou analisar suas próprias variações entre os diferentes raciocínios avaliados nos testes, com uma perspectiva intra-sujeito. Nesse contexto, a avaliação da capacidade intelectual, seja por meio da inteligência geral ou de fatores específicos, se justifica uma vez que essa tem sido demonstrada como importante fator preditivo para desempenho escolar e profissional. Não se trata, no entanto, de determinar uma profissão a partir da habilidade do sujeito, mas sim de capacitá-lo, por meio do auto-conhecimento, a explorar as possibilidades vocacionais e realizar escolhas adequadas à seus interesses, valores e outros componentes importantes para a tomada de decisão. Assim, o objetivo de avaliar a inteligência em OP seria auxiliar o sujeito a analisar seu desempenho cognitivo considerando suas experiências educacionais passadas, suas formas de aprendizagem, motivação para áreas específicas e seus projetos futuros (Almeida \& Simões, 2004).

Vale destacar que autores como Lent, Brown e Hackett (1994) e posteriormente Lent, Hackett e Brown (2004) defendem que, ao analisar os fatores que influenciam a escolha de carreira, é importante considerar, além das habilidades e interesses, outras variáveis como as expectativas de resultado, que são as crenças sobre as conseqüências da escolha, sejam elas objetivas ou subjetivas e as crenças de auto-eficácia, que envolvem a confiança do sujeito na sua capacidade para organizar e executar certas ações. Assim, num processo de OP, é necessária uma avaliação mais global de fatores sócio-cognitivos que atuam sobre as escolhas. Desse modo, a relação entre desempenho (habilidades) e interesses para a escolha de uma ocupação ou profissão não é direta, uma vez que outros fatores motivacionais interagem e podem levar a diferentes conseqüências.

Numa perspectiva empírica, buscando correlacionar habilidades e interesses, Primi e colaboradores (2002) avaliaram 60 jovens, participantes de um programa de orientação profissional. Entre os resultados, os autores observaram que os interesses (medidos pelo Levantamento de Interesses Profissionais, LIP) que se correlacionavam significativamente com inteligência (medidos pela BPR-5) foram os relacionados às áreas de Ciências Físicas (CF), Cálculo (C), Social $(\mathrm{S})$ e Lingüística (L). Mais especificamente, o Raciocínio Abstrato se relacionou positivamente com as Ciências Físicas $(r=0,40)$, Cálculo $(r=0,34)$ e negativamente com a Social $(r=-0,31)$. Já o Raciocínio Mecânico correlacionou-se com as mesmas áreas (CF: $r=0,32 ; \mathrm{C}: r=0,33$; $\mathrm{S}: r=-0,38)$, além da Lingüística, que apresentou coeficientes negativos $(r=-0,25)$. Por sua vez, o Raciocínio Espacial apresentou índices significativos com $\mathrm{C}$ $(r=0,31), \mathrm{S}(r=-0,30)$ e L $(r=-0,25)$. O Raciocínio Numérico correlacionou-se significativamente apenas com $\mathrm{C}(r=0,28)$ e, por fim, o escore geral apresentou coeficientes significativos com CF $(r=0,25), \mathrm{C}(r=0,34)$ e $\mathrm{S}(r=-0,32)$.

A partir das considerações apresentadas, o presente estudo objetivou analisar as correlações entre testes de inteligência e de interesse, utilizados em um programa de orientação vocacional. Este estudo contou com quatro instrumentos, sendo que apenas um deles possui os parâmetros psicométricos mínimos estabelecidos pelo CFP (2003), o BPR-5. Os outros instrumentos utilizados nessa pesquisa foram o INV (Forma A), o Inventário de Interesses de Angelini e o Levantamento de Interesses Profissionais. Esse estudo ganha relevância uma vez que o INV, LIP e Angelini foram instrumentos bastante ensinados a psicólogos brasileiros, de acordo com Alves, Alchieri e Marques (2002). Corroborando a observação sobre a relevância de estudar esses três testes não-aprovados pelo CFP, destaca-se o estudo de Noronha, Primi e Alchieri (2005), no qual esses testes são relatados como razoavelmente conhecidos/utilizados por psicólogos brasileiros, dentro do panorama consultado pelos autores. Mais especificamente, o INV era conhecido/utilizado por $23 \%$ da amostra, o LIP, por $21,7 \%$ e o Inventário de Interesses de Angelini, por $27 \%$.

\section{Método}

\section{Participantes}

Participaram dessa pesquisa 312 estudantes, clientes de um Programa de Orientação Profissional (OP), oferecido por uma universidade particular do interior paulista. A idade dos participantes variou entre 13 e 26 anos, com média 16,13 e desvio padrão igual a 2,07 , sendo $32,1 \%$ do sexo masculino e $67,9 \%$ do sexo feminino. O nível de escolaridade variou entre Ensino Fundamental (25,3\%) e Médio (74,7\%). A maioria desses indivíduos $(73,8 \%)$ freqüentou o programa de OP entre 7 e 10 sessões, com média de 8,31 encontros, e desvio padrão de 2,03 .

\section{Instrumentos}

Levantamento de Interesses Profissionais (LIP - DelNero, 1984). Consiste num inventário composto por 256 itens, agrupados em 128 pares de atividades profissionais, que dizem respeito a oito áreas, a saber, Ciências Físicas $(\mathrm{CF})$, Ciências Biológicas (CB), Calculísticas (C), Persuasivas (P), Administrativas (A), Sociais (S), Lingüísticas (L) e Artísticas (A). O respondente deve ler os pares e optar por um ou ambos os itens, de acordo com sua preferência. No manual, existe indicação de correção, mas não há dados normativos para interpretação.

Inventário de Interesses Angelini (Angelini, s.d.). Avalia os interesses profissionais em nove áreas: Ciências Físicas (CF); Persuasão (P); Ciências Biológicas (CB); Literatura (L); Cálculo (C); Artes (A); Música (M), Burocracia (B) e Serviços Assistenciais-Sociais (S). O instrumento é formado por 100 itens, dispostos em pares, dos quais o sujeito deve marcar apenas o que mais lhe aprouver. No manual é relatado um estudo de precisão, em que o Alfa de Cronbach variou entre 0,87 e 0,95 nas dez áreas profissionais. 
Teste de Inteligência não-verbal - Forma A (INV, Rainho, s.d.). Consiste em uma medida do fator geral de inteligência, formado por 60 itens, no qual o respondente deve descobrir e completar o padrão que governa um conjunto de figuras geométricas abstratas, dispostas em matrizes. O manual técnico relata três estudos de precisão sobre o INV. Em um, utilizou-se o método das metades, sendo que o coeficiente obtido foi de 0,82, em uma amostra de adolescentes comerciários. A precisão pelo coeficiente de Kuder-Richardson também foi obtida numa amostra de 600 crianças entre 7 e 12 anos, tendo-se obtido um alfa de 0,93. Por fim, em um estudo com 1000 adolescentes com idades entre 11 e 16 anos, os coeficientes variaram entre 0,86 e 0,96, obtidos separadamente para grupos etários.

Bateria de Prova de Raciocínio (BPR-5, Primi \& Almeida, 1998). O instrumento visa avaliar aptidões cognitivas por meio de cinco provas: raciocínio abstrato (RA-25 itens), raciocínio verbal (RV-25 itens), raciocínio mecânico (RM-25 itens), raciocínio espacial (RE- 20 itens) e raciocínio numérico (RN- 20 itens). Os participantes devem responder os problemas lógicos propostos nos itens, escolhendo entre até cinco alternativas, com exceção da prova de raciocínio numérico, que não apresenta opções de resposta pré-definidas.

Com relação aos estudos psicométricos, contou-se com uma amostra de 771 alunos de Ensino Médio e Fundamental do Estado de São Paulo. O método das metades foi aplicado e apresentou coeficiente de 0,86 . Com relação à consistência interna, as correlações de Pearson variaram entre 0,62 a 0,82 . Tais resultados atestaram uma boa precisão do instrumento. Quanto à validade de construto, a análise fatorial indicou um único fator, indicando que é capaz de avaliar uma única dimensão, ou seja, a inteligência fluída (Gf), o que é coerente com a proposta teórica que embasa o instrumento. Outros estudos de validade foram propostos desde a sua construção agrupando mais informações favoráveis, referentes a esse aspecto (Baumgartl \& Nascimento, 2004; Baumgartl \& Primi, 2006; Primi \& Almeida, 2000; Primi, Bueno, \& Muniz, 2006; Primi et al., 2002).

\section{Procedimento}

Foram realizados atendimentos coletivos, nos quais cada grupo era composto de 4 a 10 estudantes, divididos em Ensino Fundamental e Ensino Médio. Os encontros totalizaram até 12 sessões; as técnicas e instrumentos foram aplicados por estudantes de graduação de Psicologia, supervisionados por um professor responsável. A ordem de aplicação e quantidade de instrumentos utilizada por sessão foi variável, porém é importante destacar que era utilizado apenas um instrumento de inteligência e outro de interesses, ou seja, os participantes responderam BPR-5 e LIP $(n=138)$ ou o INV e o Teste de Angelini $(n=174)$. A escolha de aplicação dos conjuntos de testes foi aleatória, porém nos dois casos buscou-se fornecer informações que auxiliassem a caracterização das habilidades e interesses dos jovens, de modo a compará-las com aspectos objetivos das profissões.

\section{Resultados e discussão}

Considerando que o objetivo desse estudo envolveu a correlação entre instrumentos de interesse e de habilidades, utilizados em processos de OP, os resultados serão apresentados de modo a explicitar a relação observada. A Tabela 1 apresenta a correlação de Pearson entre os tipos de raciocínio medidos pelo BPR-5 e as áreas de interesses, avaliados pelo LIP.

Tabela 1

Coeficientes de correlação de Pearson entre os tipos de raciocínio medidos pelo BPR-5 e áreas de interesse do LIP (n $=138$ )

\begin{tabular}{|c|c|c|c|c|c|c|c|c|}
\hline \multirow{2}{*}{ BPR-5 } & \multicolumn{8}{|c|}{ Áreas de interesse do LIP } \\
\hline & $\mathrm{CF}$ & $\mathrm{CB}$ & $\mathrm{C}$ & $\mathrm{P}$ & A & $\mathrm{S}$ & $\mathrm{L}$ & A \\
\hline RA & 0,04 & 0,09 & $-0,04$ & $-0,05$ & $-0,15$ & $-0,01$ & $-0,03$ & 0,01 \\
\hline RV & $-0,14$ & 0,15 & $-0,19 *$ & $-0,05$ & $-0,25 * *$ & 0,08 & $-0,01$ & 0,12 \\
\hline RM & $0,34 * *$ & 0,11 & 0,02 & $-0,05$ & $-0,13$ & $-0,21 * *$ & $-0,24 * *$ & 0,06 \\
\hline $\mathrm{RE}$ & $0,20 * *$ & 0,03 & 0,07 & 0,00 & $-0,09$ & $-0,11$ & $-0,10$ & 0,07 \\
\hline $\mathrm{RN}$ & 0,15 & $-0,06$ & 0,09 & 0,06 & $-0,07$ & $-0,03$ & $-0,11$ & $-0,07$ \\
\hline EG & 0,16 & 0,08 & $-0,03$ & 0,01 & $-0,16$ & $-0,04$ & $-0,13$ & $-0,03$ \\
\hline
\end{tabular}

Nota: $* p<0,05 ; * * p<0,01$. RA: raciocínio abstrato, RV: raciocínio verbal, RM: raciocínio mecânico, RE: raciocínio espacial, $\mathrm{RN}$ : raciocínio numérico, EG: escore geral. CF: ciências físicas, $\mathrm{CB}$ : ciências biológicas, C: calculísticas, P: persuasivas, A: administrativas, S: sociais, L: lingüísticas, A: artísticas.

Pode-se observar que as provas do BPR que apresentaram correlações significativas foram as de raciocínio verbal (RV), mecânico (RM) e espacial (RE), resultado parcialmente diferente do que havia sido observado em outro estudo com os mesmos instrumentos (Primi et al., 2002), no qual apenas o raciocínio verbal não apresentou correlações significativas. De maneira mais específica, o presente estudo indicou correlações negativas entre o raciocínio verbal e os interesses por atividades de Cálculo e da área burocrática, ou seja, pessoas que apresentam maior extensão de vocabulário e maior profundidade do conhecimento verbal tendem a apresentar menor interesse por tarefas que envolvem o trabalho com símbolos numéricos e atividades que envolvem mais organização e repetição.

Ainda, de maneira semelhante com o encontrado por Primi et al. (2002), o raciocínio mecânico (RM) correlacionou-se positivamente com as Ciências Físicas e negativamente com os aspectos Sociais e Lingüísticos. Considerando que o RM envolve o conhecimento prático sobre problemas físico-mecânicos, parece coerente uma associação positiva com interesses na área das Ciências Físicas. Adicionalmente, um crescente raciocínio mecânico aponta para uma tendência de diminuição do envolvimento com tarefas Sociais-Assistenciais, assim como 
o uso da linguagem como principal ferramenta de trabalho. Consistentemente com o esperado, o raciocínio espacial também se correlacionou positivamente com as Ciências Físicas.

Outra comparação possível para esses resultados é com o estudo de Mansão (2005). No entanto, apesar do SDS e LIP avaliarem o mesmo construto, deve-se fazer uma ressalva de que as perspectivas teóricas utilizadas nos dois instrumentos são distintas. O manual do LIP, no entanto, conta com informações muito resumidas sobre o referencial teórico que dá base às interpretações nas áreas de interesse sugeridas, o que permite apenas uma comparação com referência aos dados empíricos. Tendo sido feita essa ressalva, pode-se mencionar que o presente resultado apresenta coerência ao ser comparado com o de Mansão (2005), que observou, ao correlacionar o SDS com as provas da BPR-5, coeficiente significativo positivo entre o RM e o tipo Realista; negativo entre RN e Social; e entre RV e Convencional. Fazendo um paralelo empírico com as áreas medidas pelo LIP, o tipo Realista pode apresentar semelhanças com as Ciências Físicas, o tipo Social de Holland com a área Social do LIP, assim como o tipo Convencional com a área burocrática, apontando para uma concordância com os resultados apresentados.

Quanto à ausência de correlações significativas (ao nível de $p<0,05$ ) entre o escore geral no BPR-5 e as áreas de interesse do LIP, o resultado contradiz os achados de Primi et al. (2002), embora tenham sido observadas correlações marginalmente significativas para as áreas das Ciências Físicas e Burocrática. Supõe-se, com essa diferença e as outras observadas em termos das correlações significativas, que o tamanho mais reduzido da amostra daquele estudo ou características específicas das amostras não identificáveis pelas variáveis sócio-demográficas disponíveis nas pesquisas possam justificar essas observações.

As correlações entre a o fator geral de inteligência, avaliado pelo INV (A) e as áreas de interesse medidas pelo Teste de Interesses de Angelini apresentaram três coeficientes significativos. Dois destes foram negativos, a saber, INV (A) com as áreas Literatura $(r=-0,210 ; p<0,01)$ e Serviços Assistenciais-Sociais $(r=-0,244 ; p<0,01)$. Isso indica uma tendência de que quanto maior o interesse por literatura e serviços assistenciais-sociais, menores os escores em inteligência geral. Por outro lado, observou-se correlação significativa positiva com Ciências Físicas $(r=0,18 ; p<0,01)$, apontando que o interesse por ciências físicas foi acompanhado por um crescimento no nível de inteligência. Faz-se importante lembrar que esses resultados não podem ser generalizados, seja porque a presente amostra não é representativa no contexto nacional, ou ainda porque foram observadas em pessoas de uma faixa etária específica. Ainda, de um modo geral pode-se levantar que a baixa correlação entre os construtos aponta para a divergência entre os mesmos, ou seja, provavelmente há pouca comunalidade entre eles, conforme já sugerido (American Educational Research Association-AERA, American Psychological Association-APA, \& National Council on Measurement in Education-NCME, 1999).

As comparações entre os resultados das correlações entre LIP e BPR-5 com INV e Angelini devem ser realizadas com cautela, uma vez que parte-se de pressupostos teóricos distintos. Uma possível comparação é entre o escore geral do BPR-5, que fornece uma medida geral de inteligência, com os escores no
INV. Nessa direção, pode-se verificar que não houve coerência, uma vez que não houve correlações significativas entre o escore geral do BPR-5 e as áreas medidas pelo LIP. Contudo, conforme já mencionado, não é possível avançar nessa discussão, sendo necessários para tanto um aprofundamento teórico que não é fornecido pelos manuais do LIP, INV e Angelini. Por fim, um comentário pertinente envolve a observação de que nos dois conjuntos de testes analisados, a magnitude das correlações observadas foi baixa, indicando a divergência entre inteligência e interesses.

\section{Considerações finais}

Esse estudo visou correlacionar instrumentos de avaliação dos interesses profissionais e de habilidades cognitivas, utilizados em processos de OP. Considerando que os três testes presentemente utilizados, não-aprovados pelo $\mathrm{CFP}$, são relatados como razoavelmente conhecidos/utilizados por psicólogos brasileiros (Noronha, Primi, \& Alchieri, 2005), torna-se importante a condução de pesquisas para que a prática profissional do psicólogo, com relação aos instrumentos, tornese baseada em critérios confiáveis e ajustadas ao seu foco de intervenção.

Ao observar correlações baixas entre os instrumentos de inteligência e interesse, reafirma-se a proposição de Lent, Brown e Hackett (1994) e de Lent, Hackett e Brown (2004), de que outras variáveis psicológicas interagem na relação entre inteligência e interesses. O presente resultado indica a divergência entre os construtos. Propõe-se que estudos futuros incluam variáveis motivacionais que auxiliem a aprofundar a discussão sobre a relação entre os construtos. Ainda, devem-se adotar delineamentos que permitam análises de dados mais sofisticadas, que extrapolem a perspectiva correlacional, buscando esclarecer os caminhos da relação entre os construtos.

Em um processo de OP, vale considerar que existe alguma relação, ainda que fraca, entre os interesses e as habilidades objetivamente medidas. Conforme destacado na literatura da área (Savickas, 1999, 2004), o orientador deve tentar incluir informações úteis para o auto-conhecimento do cliente, que possam ser comparadas com informações objetivas sobre o mercado de trabalho e as profissões. Essa meta pode ser alcançada por meio do uso conjunto de testes de interesse e de inteligência, que devem ser considerados em uma perspectiva ampla, que auxilie o sujeito a refletir sobre sua trajetória pessoal, escolar e social.

Sendo a avaliação psicológica um processo de coleta de dados sobre uma pessoa, a fim de se tomar decisões com base nesses dados, o uso de instrumentos válidos e confiáveis para tal procedimento é essencial ao psicólogo. Especialmente em processos de orientação profissional, nos quais os resultados são utilizados para facilitar as decisões que orientarão suas vidas em muitos aspectos e durante uma grande extensão de tempo, o uso de tais instrumentos deve ser baseado numa sólida base teórica e empírica, a fim de se evitar erros que possam trazer conseqüências negativas aos orientandos (AERA, APA, \& NCME, 1999).

Esse estudo sugere a realização de novos estudos com o INV, 
LIP e Angelini, de modo que eles se encontrem em condições de utilização para fins que ultrapassem os exclusivamente de pesquisa. Dessa forma, poderá haver um avanço na disponibilização de outros instrumentos para uso em OP com parâmetros de validade e precisão atestados, colaborando assim para uma atuação mais apropriada por parte dos psicólogos orientadores.

Uma das limitações do presente estudo é o fato de o mesmo ter sido realizado com jovens residentes em um único estado brasileiro. Futuros estudos poderão investigar a relação entre interesses e habilidades com outras amostras, o que ajudará na verificação de possíveis semelhanças e diferenças associadas a características específicas de grupos diferenciados. Sugerese, por fim, a formulação de novos manuais, que contenham descrições mais detalhadas dos construtos avaliados pelos testes, bem como dos estudos de validade e precisão conduzidos até então. Presentemente, a discussão dos resultados, considerando os respectivos referenciais teóricos, esteve associada a pouca informação provida pelos manuais dos testes não aprovados pelo CFP, o que reforça a importância da compilação de informações interpretativas pertinentes nos manuais de testes psicológicos.

\section{Referências}

Almeida, L. S., \& Simões, M. R. (2004). Os testes de inteligência na orientação vocacional. Em L. M. Leitão (Org.), Avaliação psicológica em orientação escolar e profissional (pp. 79-100). Coimbra: Quarteto.

Alves, I. C. B., Alchieri, J. C., \& Marques, K. C. (2002). As técnicas de exame psicológico ensinadas nos cursos de graduação de acordo com os professores. Psico-USF, 7(1), 77-88.

American Educational Research Association, American Psychological Association, \& National Council on Measurement in Education (1999). Standards for educational and psychological testing. Washington, DC: Autores.

Anastasi, A., \& Urbina, S. (2000). Testagem psicológica. Porto Alegre: Artes Médicas.

Angelini, A. L. (s.d.). Inventário de Interesses. São Paulo: CEPA.

Baumgartl, V. O., \& Nascimento, E. (2004). A Bateria de Provas de Raciocínio (BPR-5) aplicada a um contexto organizacional. Psico-USF, 9(1), 1-10.

Baumgartl, V. O., \& Primi, R. (2006). Evidências de validade da Bateria de Provas de Raciocínio (BPR-5) para seleção de pessoal. Psicologia Reflexão e Crítica, 19(2), 246-251.

Conselho Federal de Psicologia - CFP (2001). Resolução no 25/2001. Disponível em http://www.pol.org.br, acesso em 15/06/2007.

Conselho Federal de Psicologia - CFP (2003). Resolução no 02/2003. Disponível em http://www.pol.org.br, acesso em 15/06/2007.

Conselho Federal de Psicologia - CFP (2007). SATEPSI - Lista de aprovados Disponível em http://www.pol.org.br, acesso em 15/06/2007.

Crites, J. (1969). Vocational Psychology. Nova York: McGraw-Hill.

Del-Nero, C. (1984). LIP-Levantamento de Interesses Profissionais. São Paulo: Vetor

Holland, J. L. (1963). Explorations of a theory of vocational choice and achievement: a four-year prediction study. Psychological Reports, 12, 547-594.

Holland, J. L. (1977). Vocational indecision: more evidence and speculation. Journal of Counseling Psychology, 24, 404-414

Holland, J. L., Fritzsche, B. A., \& Powell, A. B. (1994). SDS - Busca Auto Dirigida (R. Primi, C. A. Biguetti, M. C. K. Pelegrini, A. M. H. Munhoz, \& E. P. D. Nucci, Trad.). Florida: Psychological Assessment Resources.

Jacquemin, A. (2000). O BBT-Br: Teste de Fotos de Profissões: normas, adaptação brasileira, estudos de caso. São Paulo: CEPA.

Lent, R., Brown, S. D., \& Hackett, G. (1994). Toward a unifying social cognitive theory of career and academic interest, choice and performance. Journal of Vocational Behavior, 45, 79-122.

Lent, R., Hackett, G., \& Brown, S. D. (2004). Una perspectiva social cognitiva de la transición entre la escuela y el trabajo. Evaluar, 4, 1-22.

Mansão, C. S. M. (2005). Interesses profissionais: validação do Self-Directed Search Career Explorer-SDS. Tese de Doutorado não-publicada, Pontifícia Universidade Católica de Campinas, Campinas, SP.

Melo-Silva, L. L., Lassance, M. C. P., \& Soares, D. H. P. (2004). A orientação profissional no contexto da educação e trabalho. Revista Brasileira de Orientação Profissional, 5(2), 31-52.

Melo-Silva, L. L., Oliveira, J. C., \& Coelho, R. S. (2002). Avaliação da orientação profissional no desenvolvimento da maturidade na escolha da profissão. PSIC - Revista de Psicologia da Vetor, 3(2), 44-53.

Neiva, K. M. C. (1999). Escala de Maturidade para Escolha Profissional (EMEP). São Paulo: Vetor.

Noronha, A. P. P., Freitas, F. A., \& Ottati, F. (2003). Análise de instrumentos de avaliação de interesses profissionais. Psicologia: Teoria e Pesquisa, 19(3), 287-291.

Noronha, A. P. P., Primi, R., \& Alchieri, J. C. (2005). Instrumentos de avaliação mais conhecidos/utilizados por psicólogos e estudantes de psicologia. Psicologia: Reflexão e Crítica, 18(5), 390-401.

Noronha, A. P., Sisto, F., \& Santos, A. A. A. (2007). Escala de Aconselhamento Profissional-EAP-Manual Técnico (Brasil). São Paulo: Vetor.

Primi, R., \& Almeida, L. S. (1998). Bateria de Provas de Raciocínio (BPR-5). Manual Técnico. São Paulo: Casa do Psicólogo.

Primi, R., \& Almeida, L. S. (2000). Estudo de validação da Bateria de Provas de Raciocínio (BPR-5). Psicologia: Teoria e Pesquisa, 16(2), 165-173.

Primi, R., Bighetti, C. A., Munhoz, A. H., Noronha, A. P. P., Polydoro, S. A J., Di Nucci, E. P., \& Pellegrini, M. C. K. (2002). Personalidade, interesses e habilidades: um estudo correlacional da BPR-5, LIP e do 16PF. Avaliação Psicológica, 1(1), 61-72.

Primi, R., Bueno, J. M. H., \& Muniz, M. (2006). Inteligência emocional: validade convergente e discriminante do MSCEIT com a BPR-5 e o 16-PF. Psicologia: Ciência e profissão, 26(1), 26-45

Rainho, O. (s.d.). Bateria Cepa: Testes de Aptidões Especificas. Rio de Janeiro: Cepa.

Savickas, M. L. (1999). Examining the personal meaning of inventoried interests during career couseling. Journal of Career Assessment, 3, 188-201.

Savickas, M. L. (2004). Um modelo para a avaliação de carreira. Em L. M. Leitão (Org.), Avaliação psicológica em orientação escolar e profissional (pp. 21-46). Coimbra: Quarteto.

Super, D. E. (1955). Dimensions and measurement of vocational maturity. Teachers College Record, 57(1), 151-163. 
Silvia Godoy, mestranda em Avaliação Psicológica pela Universidade São Francisco, é bolsista FAPESP. E-mail: silviagodoy04@yahoo.com.br

Ana Paula Porto Noronha, doutora em Psicologia como Profissão e Ciência pela Pontifícia Universidade Católica de Campinas, é docente no Programa de Pós-Graduação Stricto Sensu em Psicologia da Universidade São Francisco e bolsista de produtividade em pesquisa do CNPq. Endereço para correspondência: Rua Alexandre Rodrigues Barbosa, 45, Centro; Itatiba, S.P; CEP: 13.251-900. E-mail: ana.noronha@saofrancisco.edu.br

Rodolfo Augusto Matteo Ambiel, aluno do Curso de Psicologia da Universidade São Francisco, é bolsista de iniciação científica PIBIC/CNPq. E-mail: ambielram@yahoo.com.br

Maiana Farias Oliveira Nunes, mestre e doutoranda em Psicologia pela Universidade São Francisco, é bolsista CAPES. E-mail: maiananunes@mac.com 